\title{
The IC-Indices of Complete Bipartite Graphs
}

\author{
Chin-Lin Shiue* \\ Department of Applied Mathematics, Chung Yuan Christian University, \\ Chung Li, Taiwan 32023. email: clshiue@math.cycu.edu.tw. \\ Hung-Lin $\mathrm{Fu}^{\dagger}$ \\ Department of Applied Mathematics, National Chiao Tung University, \\ Hsin Chu, Taiwan 30050. email: hlfu@math.nctu.edu.tw. \\ Submitted: Sep 6, 2007; Accepted: Mar 5, 2008; Published: Mar 12, 2008 \\ Mathematics Subject Classifications: 05C78
}

\begin{abstract}
Let $G$ be a connected graph, and let $f$ be a function mapping $V(G)$ into $\mathbb{N}$. We define $f(H)=\sum_{v \in V(H)} f(v)$ for each subgraph $H$ of $G$. The function $f$ is called an IC-coloring of $G$ if for each integer $k$ in the set $\{1,2, \cdots, f(G)\}$ there exists an (induced) connected subgraph $H$ of $G$ such that $f(H)=k$, and the IC-index of $G$, $M(G)$, is the maximum value of $f(G)$ where $f$ is an IC-coloring of $G$. In this paper, we show that $M\left(K_{m, n}\right)=3 \cdot 2^{m+n-2}-2^{m-2}+2$ for each complete bipartite graph $K_{m, n}, 2 \leq m \leq n$.
\end{abstract}

\section{Introduction}

Given a connected graph $G$. Let $f$ be a function mapping $V(G)$ into $\mathbb{N}$. We define $f(H)=\sum_{v \in V(H)} f(v)$ for each subgraph $H$ of $G$. Then, $f$ is called an IC-coloring of $G$ if for each integer $k$ in the set $[1, f(G)]=\{1,2, \cdots, f(G)\}$ there exists an (induced) connected subgraph $H$ of $G$ such that $f(H)=k$. Clearly, the constant function $f(v)=1$ for each $v \in V(G)$ is an IC-coloring in which $f(G)=|V(G)|$. It is interesting to know the maximum value of $f(G)$, such that $f$ is an IC-coloring of $G$. This maximum value is defined as the IC-index of $G$, denoted by $M(G)$. We say that $f$ is a maximal IC-coloring of $G$ if $f$ is an IC-coloring of $G$ with $f(G)=M(G)$.

\footnotetext{
${ }^{*}$ Research supported by NSC 95-2115-M-033-005.

${ }^{\dagger}$ Research supported by NSC 95-2115-M-009-006.
} 
The study of the IC-index of a graph originated from the so-called postage stamp problem in Number Theory, which has been extensively studied in the literature $[1,6 \backsim 9$, 11, 13 16]. In 1992, G. Chappel formulated IC-colorings as "subgraph sums problem" and he observed the IC-index of cycle $C_{n}$ is bounded above by $n^{2}-n+1$, i.e., $M\left(C_{n}\right) \leq$ $n^{2}-n+1$. Later, in 1995, Penrice [12] introduced the concept of stamp covering of $G$ and he showed that (1) $M\left(K_{n}\right)=2^{n}-1$ and (2) $M\left(K_{1, n}\right)=2^{n}+2$ for all $n \geq 2$. Then, in 2005, Salehi et al proved that $M\left(K_{2, n}\right)=3 \cdot 2^{n}+1$ for $n \geq 2$ [13]. In this paper, we prove that for $2 \leq m \leq n, M\left(K_{m, n}\right)=3 \cdot 2^{m+n-2}-2^{m-2}+2$.

\section{Preliminaries}

We start with a couple of lemmas which are basic counting tools we shall use in the proof of our main result. For convenience, a sequence $c_{1}, c_{2}, \cdots, c_{n}$ of integers 0 or 1 will be referred to as a binary sequence.

Lemma 2.1. Let $a_{1}, a_{2}, \cdots, a_{n}$ be $n$ positive integers which have the properties that $a_{1}=1$ and $a_{i} \leq a_{i+1} \leq \sum_{j=1}^{i} a_{j}+1$ for $i=1,2, \cdots, n-1$. Then, for each $\ell \in\left[1, \sum_{j=1}^{n} a_{j}\right]$, there exists a binary sequence $c_{1}, c_{2}, \cdots, c_{n}$ such that $\ell=\sum_{j=1}^{n} c_{j} a_{j}$.

Proof. By induction on $n$. Clearly, it holds for $n=1$. Assume that it holds for $n=k \geq 1$. Let $\ell \in\left[1, \sum_{j=1}^{k+1} a_{j}\right]$. If $\ell \leq \sum_{j=1}^{k} a_{j}$, then by induction hypothesis, there is a binary sequence $c_{1}^{\prime}, c_{2}^{\prime}, \cdots, c_{k}^{\prime}$ such that $\ell=\sum_{j=1}^{k} c_{j}^{\prime} a_{j}$. Let $c_{j}=c_{j}^{\prime}$ for $j=1,2, \cdots, k$ and $c_{k+1}=0$. Then $\ell=\sum_{j=1}^{k+1} c_{j} a_{j}$. Otherwise, $\left(\sum_{j=1}^{k} a_{j}\right)+1 \leq \ell \leq \sum_{j=1}^{k+1} a_{j}$. Since $a_{k+1} \leq \sum_{j=1}^{k} a_{j}+1 \leq \ell$, there is an integer $\ell^{\prime} \geq 0$ such that $\ell=a_{k+1}+\ell^{\prime}$. If $\ell^{\prime}=0$, then $\ell=a_{k+1}$ and we are done. Otherwise, $1 \leq \ell^{\prime}=\ell-a_{k+1} \leq \sum_{j=1}^{k} a_{j}$. By induction hypothesis, there is a binary sequence $c_{1}^{\prime}, c_{2}^{\prime}, \cdots, c_{k}^{\prime}$ such that $\ell^{\prime}=\sum_{j=1}^{k} c_{j}^{\prime} a_{j}$. Let $c_{j}=c_{j}^{\prime}$ for $j=1,2, \cdots, k$ and $c_{k+1}=1$. Then $\ell=\ell^{\prime}+a_{k+1}=\sum_{j=1}^{k} c_{j}^{\prime} a_{j}+a_{k+1}=\sum_{j=1}^{k+1} c_{j} a_{j}$. This concludes the proof.

Lemma 2.2. Let $s_{0}, s_{1}, \cdots, s_{n}$ be a sequence of integers. Then for each $i \in[1, n]$, there exists $r_{i}$ such that $s_{i}=\sum_{j=0}^{i-1} s_{j}+r_{i}$ and the sum $\sum_{j=0}^{n} s_{j}$ is equal to $2^{n} s_{0}+\sum_{j=1}^{n} 2^{n-j} r_{j}$.

Next, we explore several necessary conditions for the existence of an IC-coloring of a graph $G$. Without mention otherwise, all graphs we consider in what follows are connected. For graph terms, we refer to [17].

Lemma 2.3. Let $f$ be an IC-coloring of a graph $G$ such that $f\left(u_{i}\right) \leq f\left(u_{i+1}\right)$ for $i=1,2, \cdots, n-1$, where $V(G)=\left\{u_{1}, u_{2}, \cdots, u_{n}\right\}$. Then $f\left(u_{1}\right)=1$ and $f\left(u_{i+1}\right) \leq$ $\sum_{j=1}^{i} f\left(u_{j}\right)+1$ for $i=1,2, \cdots, n-1$.

Proof. Clearly, $f\left(u_{1}\right)=1$. Suppose that $f\left(u_{i+1}\right)>\sum_{j=1}^{i} f\left(u_{j}\right)+1$ for some $i \in[1, n-1]$ and $H$ is a subgraph of $G$ with $f(H)=\sum_{j=1}^{i} f\left(u_{j}\right)+1$. By the assumption, $f\left(u_{j}\right)>f(H)$ for each $j \in[i+1, n]$. This implis that $V(H) \subseteq\left\{u_{1}, u_{2}, \cdots, u_{i}\right\}$ and we have $f(H) \leq$ $\sum_{j=1}^{i} f\left(u_{j}\right)$. Hence, we have a contradiction and the proof is complete. 
Lemma 2.4. Let $f$ be an IC-coloring of a graph $G$ such that $f\left(u_{i}\right)<f\left(u_{i+1}\right)$ for $i=$ $1,2, \cdots, n-1$, where $V(G)=\left\{u_{1}, u_{2}, \cdots, u_{n}\right\}$. For each pair $i_{1}, i_{2}, 1 \leq i_{1}<i_{2} \leq n$, if $f\left(u_{i_{1}}\right)=\sum_{j=1}^{i_{1}-1} f\left(u_{j}\right)+1$ and $u_{i_{1}} u_{i_{2}} \notin E(G)$, then either $f\left(u_{i_{2}}\right) \leq \sum_{j=1}^{i_{2}-1} f\left(u_{j}\right)-f\left(u_{i_{1}}\right)$ or $f\left(u_{i_{2}+1}\right) \leq f\left(u_{i_{1}}\right)+f\left(u_{i_{2}}\right)$ when $i_{2}+1 \leq n$.

Proof. Suppose, to the contrary, $f\left(u_{i_{2}}\right)>\sum_{j=1}^{i_{2}-1} f\left(u_{j}\right)-f\left(u_{i_{1}}\right)$ and $f\left(u_{i_{2}+1}\right)>f\left(u_{i_{1}}\right)+$ $f\left(u_{i_{2}}\right)$ when $i_{2}+1 \leq n$. Let $k=f\left(u_{i_{1}}\right)+f\left(u_{i_{2}}\right)$ and let $H$ be an induced connected subgraph of $G$ such that $f(H)=k$. By the assumption, $f\left(u_{i}\right) \geq f\left(u_{i_{2}+1}\right)>k$ for each $i \in\left[i_{2}+1, n\right]$. This implies that $V(H) \subseteq\left\{u_{1}, u_{2}, \cdots, u_{i_{2}}\right\}$. Also by the assumption, it is easy to see $u_{i_{2}} \in V(H)$. Since $f(H)=f\left(u_{i_{1}}\right)+f\left(u_{i_{2}}\right)$ and $\left\{u_{i_{1}}, u_{i_{2}}\right\}$ is an independent set, $u_{i_{1}} \notin V(H)$. If $u_{j} \notin V(H)$ for each $j \in\left[i_{1}+1, i_{2}-1\right]$, then by the hypothesis, we have $f(H) \leq f\left(u_{2}\right)+\sum_{j=1}^{i_{1}-1} f\left(u_{j}\right)<f\left(u_{i_{2}}\right)+f\left(u_{i_{1}}\right)=k$, a contradiction. Otherwise, $u_{j} \in V(H)$ for some $j \in\left[i_{1}+1, i_{2}-1\right]$. This implies $f(H) \geq f\left(u_{i_{2}}\right)+f\left(u_{j}\right)>f\left(u_{i_{2}}\right)+f\left(u_{i_{1}}\right)=k$, a contradiction. Therefore, we have the proof.

The following facts are useful in proving our main result.

Lemma 2.5. Let $r_{1}, r_{2}, \cdots, r_{n}$ be $n$ numbers. If there are two integers $i$ and $k$ such that $1 \leq i<k \leq n$ and $r_{i}<r_{k}$, then $\sum_{j=1}^{n} 2^{n-j} r_{j}<\sum_{j=1}^{n} 2^{n-j} r_{j}-\left(2^{n-i} r_{i}+2^{n-k} r_{k}\right)+\left(2^{n-k} r_{i}+\right.$ $\left.2^{n-i} r_{k}\right)$.

Lemma 2.6. Let $f$ be an $I C$-coloring of a graph $G$, and let $G$ has $\ell$ induced connected subgraphs. If there are $2 k$ distinct induced connected subgraphs $H_{1}, G_{1}, H_{2}, G_{2}, \cdots, H_{k}$, $G_{k}$ of $G$ such that $f\left(H_{i}\right)=f\left(G_{i}\right)$ for $i=1,2, \cdots, k$, then $f(G) \leq \ell-k$.

Now, we are ready for the main result.

\section{Main Result}

First, we establish the lower bound of $M\left(K_{m, n}\right)$.

Proposition 3.1. $M\left(K_{m, n}\right) \geq 3 \cdot 2^{m+n-2}-2^{m-2}+2$ for $2 \leq m \leq n$.

Proof. Let $G=(A, B)=K_{m, n}, 2 \leq m \leq n$, with vertex sets $A=\left\{a_{1}, a_{2}, \cdots, a_{m}\right\}$ and $B=\left\{b_{1}, b_{2}, \cdots, b_{n}\right\}$, and let $f: V(G) \rightarrow \mathbb{N}$ be defined by (see Figure 1 for an example):

(i) $f\left(a_{1}\right)=1, f\left(a_{2}\right)=2$ and $f\left(b_{1}\right)=3$;

(ii) $f\left(b_{i}\right)=f\left(a_{1}\right)+f\left(a_{2}\right)+\sum_{j=1}^{i-1} f\left(b_{j}\right)$ for $i=2,3, \cdots, n-1$;

(iii) $f\left(b_{n}\right)=\left[f\left(a_{1}\right)+f\left(a_{2}\right)+\sum_{j=1}^{n-1} f\left(b_{j}\right)\right]+1$; and

(iv) $f\left(a_{i}\right)=f\left(a_{1}\right)+f\left(a_{2}\right)+\sum_{j=1}^{n} f\left(b_{j}\right)+\sum_{j=3}^{i-1} f\left(a_{j}\right)-2$ for $i=3,4, \cdots, m$.

First, we evaluate $f(G)$. Let $s_{0}, s_{1}, \cdots, s_{m+n-2}$ be a sequence defined by 


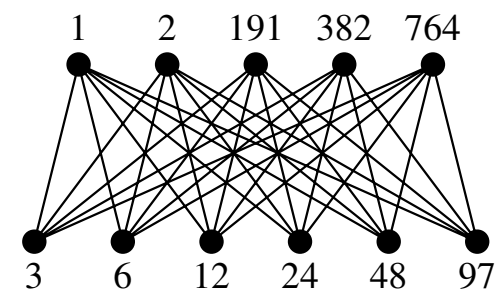

Figure 1: An IC-coloring of $K_{5,6}$

(a) $s_{0}=f\left(a_{1}\right)+f\left(a_{2}\right)=3$;

(b) $s_{i}=f\left(b_{i}\right)$ for $i=1,2, \cdots, n$; and

(c) $s_{n+i}=f\left(a_{i+2}\right)$ for $i=1,2, \cdots, m-2$.

Then, we have $s_{i}=\sum_{j=0}^{i-1} s_{j}+0$ for $i=1,2, \cdots, n-1, s_{n}=\sum_{j=0}^{n-1} s_{j}+1$, and $s_{i}=$ $\sum_{j=0}^{i-1} s_{j}-2$ for $i=n+1, n+2, \cdots, m+n-2$. By Lemma 2.2, we have

$$
\begin{aligned}
f(G) & =\sum_{j=1}^{m} f\left(a_{j}\right)+\sum_{j=1}^{n} f\left(b_{j}\right) \\
& =\sum_{j=0}^{m+n-2} s_{j}=2^{m+n-2} s_{0}+\sum_{j=n+1}^{m+n-2} 2^{(m+n-2)-j}(-2)+1 \cdot 2^{(m+n-2)-n} \\
& =3 \cdot 2^{m+n-2}-2\left(2^{m-2}-1\right)+2^{m-2}=3 \cdot 2^{m+n-2}-2^{m-2}+2 .
\end{aligned}
$$

It is left to show that $f$ is an IC-coloring of $G$. For convenience, we rename the vertices of $G$ to be $u_{1}, u_{2}, \cdots, u_{m+n}$ such that $f\left(u_{1}\right)<f\left(u_{2}\right)<\cdots<f\left(u_{m+n}\right)$. By the definition of $f$, we have $f\left(u_{1}\right)=f\left(a_{1}\right)=1$ and $f\left(u_{i}\right)<f\left(u_{i+1}\right) \leq \sum_{j=1}^{i} f\left(u_{j}\right)+1$ for $i=1,2, \cdots, m+n-1$. Then, by Lemma 2.1 , for each $k \in\left[1, \sum_{j=1}^{m+n} f\left(u_{j}\right)\right]=[1, f(G)]$, there is a binary sequence $c_{1}, c_{2}, \cdots, c_{m+n}$ such that $k=\sum_{j=1}^{m+n} c_{j} f\left(u_{j}\right)$.

Now, we will prove that there is an induced connected subgraph $H$ of $G$ such that $f(H)=k=\sum_{j=1}^{m+n} c_{j} f\left(u_{j}\right)$. Let $S=\left\{u_{j} \mid c_{j}=1\right.$ and $\left.j \in[1, m+n]\right\}$. Clearly, we have $f\left(<S>_{G}\right)=k$ where $<S>_{G}$ is the induced subgraph of $G$ induced by $S$. Since $k>0$, $S$ is nonempty. If $\langle S\rangle_{G}$ is connected, then $H=\langle S\rangle_{G}$ is desired. Otherwise, $\langle S\rangle_{G}$ is disconnected and thus $S$ is an independent set of size at least two. Since $G=(A, B)$ is a complete bipartite graph, we also have $S \subseteq A$ or $S \subseteq B$ but not both. Note that $A=\left\{u_{1}, u_{2}\right\} \bigcup\left\{u_{j} \mid j \in[n+3, m+n]\right\}$ and $B=\left\{u_{j} \mid j \in[3, n+2]\right\}$ by the definition of $f$. To complete the proof, we consider the following four cases.

Case 1. $\left\{u_{1}, u_{2}\right\} \subseteq S \subseteq A$.

Let $S_{1}=\left(S \backslash\left\{u_{1}, u_{2}\right\}\right) \cup\left\{u_{3}\right\}$ and let $H=\left\langle S_{1}>_{G}\right.$. Then, $H$ is connected and $f(H)=k-f\left(u_{1}\right)-f\left(u_{2}\right)+f\left(u_{3}\right)=k$. 
Case 2. $u_{1} \in S, u_{2} \notin S$ and $S \subseteq A$

Let $\ell=\min \left\{j \mid c_{j}=1\right.$ and $\left.j \geq n+3\right\}$. Then by the definition of $f$, we have $f\left(u_{\ell}\right)=$ $\sum_{j=1}^{\ell-1} f\left(u_{j}\right)-2$. This implies that $f\left(u_{\ell}\right)+f\left(u_{1}\right)=\sum_{j=2}^{\ell-1} f\left(u_{j}\right)+2 f\left(u_{1}\right)-2=\sum_{j=2}^{\ell-1} f\left(u_{j}\right)$. Let $S_{1}=\left(S \backslash\left\{u_{1}, u_{\ell}\right\}\right) \cup\left\{u_{j} \mid j \in[2, \ell-1]\right\}$, and let $H=\left\langle S_{1}>_{G}\right.$. Then, $H$ is connected and $f(H)=k-\left(f\left(u_{1}\right)+f\left(u_{\ell}\right)\right)+\sum_{j=2}^{\ell-1} f\left(u_{j}\right)=k$.

Case 3. $u_{1} \notin S$ and $S \subseteq A$.

Let $\ell=\min \left\{j \mid c_{j}=1\right.$ and $\left.j \geq n+3\right\}$. Then $f\left(u_{\ell}\right)=\sum_{j=1}^{\ell-1} f\left(u_{j}\right)-2=\sum_{j=1}^{\ell-1} f\left(u_{j}\right)-$ $f\left(u_{2}\right)=f\left(u_{1}\right)+\sum_{j=3}^{\ell-1} f\left(u_{j}\right)$. Let $S_{1}=\left(S \backslash\left\{u_{\ell}\right\}\right) \cup\left\{u_{1}, u_{3}, u_{4}, \cdots, u_{\ell-1}\right\}$, and $H=<S_{1}>_{G}$. Then, $H$ is connected and $f(H)=k-f\left(u_{\ell}\right)+f\left(u_{1}\right)+\sum_{j=3}^{\ell-1} f\left(u_{j}\right)=k$.

Case 4. $S \subseteq B$.

Let $\ell=\min \left\{j \mid c_{j}=1\right.$ and $\left.j \geq 3\right\}$. Since $|S| \geq 2$, we have $3 \leq \ell \leq n-1$. By the definition of $f, f\left(u_{\ell}\right)=\sum_{j=1}^{\ell-1} f\left(u_{j}\right)$. Let $S_{1}=\left(S \backslash\left\{u_{\ell}\right\}\right) \cup\left\{u_{1}, u_{2}, \cdots, u_{\ell-1}\right\}$. Then, $H=$ $<S_{1}>_{G}$ is connected and $f(H)=k-f\left(u_{\ell}\right)+\sum_{j=1}^{\ell-1} f\left(u_{j}\right)=k$. This concludes the proof.

We remark here that we intend to prove that $M\left(K_{m, n}\right)$ is equal to the lower bound obtained in Proposition 3.1. Therefore, we shall prove that the lower bound is also the upper bound. First, we estimate the number of induced connected subgraphs of $K_{m, n}$.

Proposition 3.2. $K_{m, n}$ has $2^{m+n}-\left(2^{m}+2^{n}\right)+(m+n+1)$ induced connected subgraphs.

Proof. Let $G=(A, B)=K_{m, n}$. For any induced connected subgraph $H$, either $|V(H)|=$ 1 or $V(H) \cap A \neq \phi$ and $V(H) \cap B \neq \phi$. Therefore, the number of induced connected subgraphs of $K_{m, n}$ is equal to $(m+n)+\left(2^{m}-1\right)\left(2^{n}-1\right)$.

Note that the number of distinct induced connected subgraphs of $G$ does provide a natural upper bound for $M(G)$. But, after an IC-coloring is given, we may have distinct induced connected subgraphs which receive common values and thus the upper bound will be smaller. In what follows, we obtain several properties of a maximal IC-coloring $f$ of $K_{m, n}$.

Proposition 3.3. If $f$ is a maximal IC-coloring of $G=(A, B)=K_{m, n}$, then all the colorings of vertices of $G$ are distinct.

Proof. Suppose, to the contrary, there exist two distinct vertices $u$ and $v$ such that $f(u)=$ $f(v)$. Now, depending on the distribution of $u$ and $v$ in $A \cup B$, we have three cases to consider: (1) $u \in A$ and $v \in B$, (2) $u, v \in A$ and (3) $u, v \in B$. Observe that if there exists a set $S \subseteq(A \cup B) \backslash\{u, v\}$ such that $H_{1}=\langle S \cup\{u\}\rangle_{G}$ and $H_{2}=\langle S \cup\{v\}\rangle_{G}$ are two induced connected subgraphs of $G$, then $f\left(H_{1}\right)=f\left(H_{2}\right)$. Therefore, the number $\alpha$ of such subsets $S$ gives the number of graph pairs which have the same function value. Then, by Lemma 2.6 and Proposition 3.2, we conclude that $f(G) \leq 2^{m+n}-\left(2^{m}+2^{n}\right)+(m+n+1)-\alpha$. So, $\alpha$ determines the upper bound of $f(G)$. 
By direct counting, it is not difficult to see that there are $\left(2^{m-1}-1\right)\left(2^{n-1}-1\right)+1$, $2^{m-2}\left(2^{n}-1\right)+1$ and $\left(2^{m}-1\right) 2^{n-2}+1$ subsets $S$ for the above three cases respectively to produce graph pairs with the same function value. Thus,

$$
\begin{aligned}
f(G) & \leq 2^{m+n}-\left(2^{m}+2^{n}\right)+(m+n+1) \\
& -\min \left\{\left(2^{m-1}-1\right)\left(2^{n-1}-1\right)+1,2^{m-2}\left(2^{n}-1\right)+1,\left(2^{m}-1\right) 2^{n-2}+1\right\} \\
& =2^{m+n}-\left(2^{m}+2^{n}\right)+(m+n+1)-\left(2^{m+n-2}-2^{m-1}-2^{n-1}+2\right) \\
& <3 \cdot 2^{m+n-2}-2^{m-2}+2 .
\end{aligned}
$$

Hence, by Proposition 3.1, $f$ is not a maximal IC-coloring, a contradiction. This concludes the proof.

Now, we let $G=(A, B)=K_{m, n}, 2 \leq m \leq n$, and let $f$ be a maximal IC-coloring of $G$. By Proposition 3.3, we may let $f\left(u_{i}\right)<f\left(u_{i+1}\right)$ for $i=1,2, \cdots, m+n-1$. where $V(G)=\left\{u_{1}, u_{2}, \cdots, u_{m+n}\right\}$. For convenience, we also define $f_{i}=\sum_{j=1}^{i} f\left(u_{j}\right)$ for $i=1,2, \cdots, m+n$. The following proposition is essential to the proof of the main theorem.

Proposition 3.4. Let $f$ be a maximal $I C$-coloring of $G=K_{m, n}$. Then, we have

(1) $f\left(u_{1}\right)=1, f\left(u_{2}\right)=2$ and $f\left(u_{3}\right)=3$ or 4 , moreover, $f\left(u_{3}\right)=3$ if $u_{1} u_{2} \notin E(G)$.

(2) $f_{4} \leq 13$ and equality holds only if $<\left\{u_{1}, u_{2}, u_{3}, u_{4}\right\}>_{G} \cong K_{2,2}$.

(3) If $j \in[5, m+n]$ and $u_{j} u_{t} \notin E(G)$ where $t=1$ or 2 , then $f\left(u_{j}\right) \leq f_{j-1}-t$.

(4) $f_{j}>3 \cdot 2^{j-2}-2^{j-(n+2)}-1$ for each $j \in[1, m+n]$.

Proof. The conclusion of $(1)$ and $f_{4} \leq 13$ are easy to see, we assume that $f_{4}=13$. We claim that $H=\left\langle\left\{u_{1}, u_{2}, u_{3}, u_{4}\right\}\right\rangle_{G} \cong K_{2,2}$. First, we need an inequality.

For each $i \in[1, m+n]$ and each $j \in[i, m+n], f_{j}<2^{j-i}\left(f_{i}+1\right)$

By Lemma 2.3, we have $f\left(u_{i+k}\right) \leq f_{i+k-1}+1=f_{i}+\sum_{\ell=1}^{k-1} f\left(u_{i+\ell}\right)+1$ for each $k \in[1, m+n-i]$. Then considering the sequence $f_{i}, f\left(u_{i+1}\right) \cdots, f\left(u_{j}\right)$ in Lemma 2.2, we obtain

$$
f_{j}=\sum_{k=1}^{j} f\left(u_{k}\right)=f_{i}+\sum_{k=1}^{j-i} f\left(u_{i+k}\right) \leq 2^{j-i} f_{i}+\left(2^{j-i}-1\right)<2^{j-i}\left(f_{i}+1\right) .
$$

Therefore, we have $(*)$. Now, we are ready for the proof of $(2)$.

Note that $f\left(u_{i_{1}}\right)=\sum_{j=1}^{i_{1}-1} f\left(u_{j}\right)+1$ for $i_{1}=1$ or 2 . Suppose that $f_{4}=13$ and $H$ is not isomorphic to $K_{2,2}$. Since $G$ is a complete bipartite graph, $H$ is ismorphic to either $K_{1,3}$ or $I_{4}$ (an independent set of size 4 ). 
Case 1. $u_{1} u_{2} \notin E(G)$.

By (1), $f\left(u_{3}\right)=3>f_{2}-f\left(u_{1}\right)$. If $\left\{u_{1}, u_{2}, u_{3}\right\}$ is an independent set, then $f\left(u_{4}\right) \leq$ $f\left(u_{3}\right)+f\left(u_{1}\right)=4$ by Lemma 2.4. This contradicts to the assumption that $f_{4}=13$. Hence, $u_{3} u_{1} \in E(G)$ and $u_{3} u_{2} \in E(G)$. By the assumption, $f\left(u_{4}\right)=7>f_{3}-f\left(u_{1}\right)$ and $\left\{u_{1}, u_{2}, u_{4}\right\}$ is an independent set. If $m+n=4$, then we have a contradiction to that $f$ is an IC-coloring of $G$ by Lemma 2.4. Otherwise, $m+n \geq 5$ and hence $n \geq 3$. By Lemma 2.4, $f\left(u_{5}\right) \leq f\left(u_{1}\right)+f\left(u_{4}\right)=8$. This implies that $f_{5} \leq 21$. Hence, by $(*)$, we have

$$
\begin{aligned}
f(G) & <2^{m+n-5}\left(f_{5}+1\right)=2^{m+n-5} \cdot 22<24 \cdot 2^{m+n-5}-2^{m+n-5} \\
& \leq 3 \cdot 2^{m+n-2}-2^{m-2}+2 .
\end{aligned}
$$

By Proposition 3.1, it contradicts to that $f$ is a maximal IC-coloring of $G$.

Case 2. $u_{1} u_{2} \in E(G)$.

Since $G$ is a complete bipartitle graph, either $u_{3} u_{1} \notin E(G)$ or $u_{3} u_{2} \notin E(G)$. If $u_{1} u_{3} \notin E(G)$, we have $f\left(u_{4}\right) \leq f\left(u_{1}\right)+f\left(u_{3}\right) \leq 5$ by (1) and Lemma 2.4. This implies that $f_{4} \leq 12$. This is a contradiction to our assumption. Hence, $u_{2} u_{3} \notin E(G)$ and so $f\left(u_{4}\right) \leq f\left(u_{2}\right)+f\left(u_{3}\right) \leq 6$ by (1) and Lemma 2.4. Also, by our assumption, it is easy to see that $f\left(u_{3}\right)=4, f\left(u_{4}\right)=6>f_{3}-f\left(u_{2}\right)$ and $\left\{u_{2}, u_{3}, u_{4}\right\}$ is an independent set. By Lemma 2.4, $f\left(u_{5}\right) \leq f\left(u_{2}\right)+f\left(u_{4}\right)=8$. This implies that $f_{5} \leq 21$. Then by $(*)$ in Case 1 , we have $f(G)<3 \cdot 2^{m+n-2}-2^{m-2}+2$, a contradiction. Hence, (2) is proved. Next, we prove (3). Since $t=2$ is a similar case, we prove the case $t=1$.

Suppose, to the contrary, that $f\left(u_{j}\right)>f_{j-1}-1=f_{j-1}-f\left(u_{1}\right)$ for some $j \in[5, m+n]$. Then by Lemma 2.4, if $j=m+n$, then $f$ is not an IC-coloring of $G$ and we are done. Otherwise, $f\left(u_{j+1}\right) \leq f\left(u_{j}\right)+f\left(u_{1}\right)=f\left(u_{j}\right)+1$. By $(*)$, we have

$$
f_{j} \leq 2^{j-4}\left(f_{4}+1\right)-1=14 \cdot 2^{j-4}-1 \text { and } f_{j-1} \leq 2^{j-5}\left(f_{4}+1\right)-1 \leq 14 \cdot 2^{j-5}-1 .
$$

This implies that

$$
\begin{aligned}
f_{j+1} & =f_{j}+f\left(u_{j+1}\right) \leq f_{j}+f\left(u_{j}\right)+1 \leq f_{j}+f_{j-1}+2 \\
& \leq\left(14 \cdot 2^{j-4}-1\right)+\left(14 \cdot 2^{j-5}-1\right)+2=21 \cdot 2^{j-4} .
\end{aligned}
$$

Since $j \geq 5$ and $n \geq 2$, we have

$$
\begin{aligned}
f(G) & <2^{m+n-(j+1)}\left(f_{j+1}+1\right) \leq 2^{m+n-(j+1)}\left(22 \cdot 2^{j-4}\right) \\
& =3 \cdot 2^{m+n-2}-2^{m+n-4}<3 \cdot 2^{m+n-2}-2^{m-2}+2 .
\end{aligned}
$$


By Proposition 3.1, it contradicts to the assumption that $f$ is a maximal IC-coloring of $G$ and we have the proof of (3). Finally, we prove (4).

Suppose that $f_{j} \leq 3 \cdot 2^{j-2}-2^{j-(n+2)}-1$ for some $j \in[1, m+n]$. Then by $(*)$, we have

$$
\begin{aligned}
f(G) & =f_{m+n}<2^{m+n-j}\left(f_{j}+1\right) \leq 2^{m+n-j}\left(3 \cdot 2^{j-2}-2^{j-(n+2)}\right) \\
& <3 \cdot 2^{m+n-2}-2^{m-2}+2 .
\end{aligned}
$$

This is a contradiction and we have the proof of (4).

Theorem 3.5. $M\left(K_{m, n}\right)=3 \cdot 2^{m+n-2}-2^{m-2}+2$ for $2 \leq m \leq n$.

Proof. Let $G=K_{m, n}, V(G)=\left\{u_{1}, u_{2}, \cdots, u_{m+n}\right\}$ and $f$ be a maximal IC-coloring of $G$. Since $M\left(K_{m, n}\right) \geq 3 \cdot 2^{m+n-2}-2^{m-2}+2$ by Proposition 3.1, it sufficies to show that $M\left(K_{m, n}\right) \leq 3 \cdot 2^{m+n-2}-2^{m-2}+2$. From (2) of Proposition 3.4, it is true for $m=n=2$. So, assume that $n \geq 3$. By Proposition 3.3, we may let $f\left(u_{i}\right)<f\left(u_{i+1}\right)$ for $i \in[1, m+n-1]$. For convenience of calculation, we also let $f_{i}=\sum_{\ell=1}^{i} f\left(u_{\ell}\right)$ for $i \in[1, m+n]$ and $f\left(u_{i+\ell}\right)=f_{i+\ell-1}+r_{\ell}$ for $\ell \in[1, m+n-i]$. By Lemma 2.3, we have $r_{\ell} \leq 1$ for $\ell \in[1, m+n-i]$. Now, by Lemma 2.2, we have

$$
f_{i+j}=\sum_{\ell=1}^{i+j} f\left(u_{\ell}\right)=f_{i}+\sum_{\ell=1}^{j} f\left(u_{i+\ell}\right)=2^{j} f_{i}+\sum_{\ell=1}^{j} 2^{j-\ell} r_{\ell} .
$$

This implies that (by letting $i=4$ )

$$
f(G)=f_{m+n}=f_{4+(m+n-4)}=2^{m+n-4} f_{4}+\sum_{\ell=1}^{m+n-4} 2^{m+n-4-\ell} r_{\ell} .
$$

First, if $u_{1} u_{2} \in E(G)$, then either $u_{4+\ell} u_{1} \notin E(G)$ or $u_{4+\ell} u_{2} \notin E(G)$ (but not both) for each $\ell \in[1, m+n-4]$. Since $f$ is a maximal coloring, by (3) of Proposition 3.4. we have $f\left(u_{j}\right) \leq f_{j-1}-t$ provided $j \in[5, m+n]$ and $u_{j} u_{t} \notin E(G)$ where $t=1$ or 2. This implies $r_{\ell} \leq-1$ or -2 depending on $t=1$ or 2 . Thus, by $\left(*^{\prime \prime}\right)$, we have $f(G) \leq 2^{m+n-4} \cdot f_{4}+\sum_{\ell=1}^{m+n-4} 2^{m+n-4-\ell} \cdot(-1)$. Now, in case that $f_{4} \leq 12, f(G) \leq$ $3 \cdot 2^{m+n-2}-\left(2^{m+n-4}-1\right) \leq 3 \cdot 2^{m+n-2}-2^{m-2}+2$. On the other hand, $f_{4}=13$ and the graph $H$ induced by $\left\langle\left\{u_{1}, u_{2}, u_{3}, u_{4}\right\}\right\rangle_{G}$ is isomorphic to $K_{2,2}$ by (2) of Proposition 3.4. Clearly, there are $m-2$ vertices in one partite set of $G-H$ and $n-2$ vertices in the other partite set. Therefore, since $n-2 \geq m-2$,

$$
\begin{aligned}
f(G) & \leq 2^{m+n-4} \cdot f_{4}+\sum_{j=1}^{n-2} 2^{m+n-4-j} \cdot(-1)+\sum_{j=n-1}^{m+n-4} 2^{(m+n-4)-j} \cdot(-2)\left(\text { by }\left(*^{\prime \prime}\right)\right) \\
& =13 \cdot 2^{m+n-4}+(-1)\left[2^{m+n-4}-1\right]+(-1)\left[2^{m-2}-1\right] \\
& =3 \cdot 2^{m+n-2}-2^{m-2}+2 .
\end{aligned}
$$

Hence, we have the proof. In what follows, we assume that $u_{1} u_{2} \notin E(G)$. By (1) and (2) of Proposition 3.4, we have $f\left(u_{3}\right)=3$ and $4 \leq f\left(u_{4}\right) \leq 7$. If $f\left(u_{4}\right)=4$, then since 
$n \geq 3$, we have $f_{4}=10 \leq 3 \cdot 2^{4-2}-2^{4-(n+2)}-1$. This is a contradiction to that $f$ is a maximal IC-coloring of $G$ by (4) of Proposition 3.4. Hence, $5 \leq f\left(u_{4}\right) \leq 7$. First, we claim that $H$ is isomorphic to $K_{2,2}$. Suppose not. If $\left\{u_{1}, u_{2}, u_{3}\right\}$ is an independent set, then by Lemma 2.4 and $f\left(u_{3}\right)=3>f_{2}-f\left(u_{1}\right)$, we have $f\left(u_{4}\right) \leq f\left(u_{1}\right)+f\left(u_{3}\right)=4$, a contradiction. Hence, $u_{3}$ is adjacent to $u_{1}$ and $u_{2}$. Thus, $\left\{u_{1}, u_{2}, u_{4}\right\}$ must be an independent set. Since $f\left(u_{4}\right) \geq 5>4=f_{3}-f\left(u_{2}\right)$, we have $f\left(u_{5}\right) \leq f\left(u_{2}\right)+f\left(u_{4}\right) \leq 9$ by Lemma 2.4. This implies $f_{5} \leq 22 \leq 3 \cdot 2^{5-2}-2^{5-(n+2)}-1$ and we have a contradiction by (4) of proposition 3.4. So, $H \cong K_{2,2}=(A, B)$ where $A=\left\{u_{1}, u_{2}\right\}$ and $B=\left\{u_{3}, u_{4}\right\}$. Now, let $V_{1}=\left\{v \in V(G) \backslash V(H) \mid v u_{2} \notin E(G)\right\}$ and $V_{2}=\left\{v \in V(G) \backslash V(H) \mid v u_{4} \notin E(G)\right\}$. Then $\left\{V_{1}, V_{2}\right\}$ is a partition of $V(G) \backslash V(H)$ such that $\left|V_{1}\right|=n-2,\left|V_{2}\right|=m-2$ or $\left|V_{1}\right|=m-2$, $\left|V_{2}\right|=n-2$. Then by (3) of Proposition 3.4, $r_{\ell} \leq-2$ in $\left(*^{\prime \prime}\right)$ if $u_{4+\ell} \in V_{1}$. Now, the proof follows by considering the following two cases.

Case $1.5 \leq f\left(u_{4}\right) \leq 6$.

Clearly, we have $f_{4} \leq 12$. If for each $u_{k} \in V_{2}, f\left(u_{k}\right) \leq f_{k-1}$, then $r_{\ell} \leq 0$ provided that $u_{4+\ell} \in V_{2}$ in $\left(*^{\prime \prime}\right)$. By Lemma 2.5 and $\left(*^{\prime \prime}\right)$, we have

$$
\begin{aligned}
f(G) & \leq 2^{m+n-4} f_{4}+\sum_{j=1}^{n-2} 2^{m+n-4-j} \cdot 0+\sum_{j=n-1}^{m+n-4} 2^{m+n-4-j} \cdot(-2) \\
& =2^{m+n-4} \cdot 12+\left(2^{m-2}-1\right) \cdot(-2) \leq 3 \cdot 2^{m+n-2}-2^{m-2}+2 .
\end{aligned}
$$

Otherwise, there exists a $u_{k} \in V_{2}$ such that $f\left(u_{k}\right)=f_{k-1}+1$. Let $i$ be the smallest integer such that $f\left(u_{i}\right)=f_{i-1}+1$ and $u_{i} \in V_{2}$. Then for each $k \in[5, i-1], r_{k-4} \leq 0$ in $\left(*^{\prime}\right)$. This implies that $f_{i-1}=f_{4+(i-5)} \leq 2^{i-5} f_{4} \leq 3 \times 2^{i-3}$. Again, by (4) of Proposition 3.4, we have $f_{i-1}>3 \cdot 2^{i-3}-2^{i-(n+3)}-1$. This implies that

$$
3 \cdot 2^{i-3}-2^{i-(n+3)}<f\left(u_{i}\right) \leq 3 \times 2^{i-3}+1 .
$$

Moreover, let $\left|V_{2}\right|=t$. We claim that $i=\max \left\{k \mid v_{k} \in V_{2}\right\}$. Suppose not. Let $j$ be the smallest positive integer such $u_{i+j} \in V_{2}$. Then $i+j \leq m+n$ and by Lemma 2.4, either $f\left(u_{i+j}\right) \leq f_{i+j-1}-f\left(u_{i}\right)$ or $f\left(u_{i+j+1}\right) \leq f\left(u_{i}\right)+f\left(u_{i+j}\right)$ when $i+j+1 \leq m+n$.

First, if $f\left(u_{i+j}\right) \leq f_{i+j-1}-f\left(u_{i}\right)$, then, for $j=1, f\left(u_{i+1}\right) \leq f_{i}-f\left(u_{i}\right)=f_{i-1}<$ $f\left(u_{i}\right)$. This contradicts to the definition of $f$. Hence $j \geq 2$. This implies that for $k \in[i+1, i+j-1], u_{k} \in V_{1}$ and $r_{k-i} \leq-2$ in $\left(*^{\prime}\right)$ by (3) of Proposition 3.4. Therefore, by Lemma $2.5,\left(*^{\prime}\right)$ and $(* *)$,

$$
\begin{aligned}
f_{i+j-1} & \leq 2^{j-1} \cdot f_{i}+\sum_{\ell=1}^{j-1} 2^{(j-1)-\ell} \cdot(-2)=2^{j-1}\left[f_{i-1}+f\left(u_{i}\right)\right]-2\left(2^{j-1}-1\right) \\
& \leq 2^{j-1}\left(3 \cdot 2^{i-2}+1\right)-2\left(2^{j-1}-1\right)=3 \cdot 2^{i+j-3}-2^{j-1}+2 . \ldots \ldots \ldots
\end{aligned}
$$

Again, by $(* *),\left(* *^{\prime}\right)$ and the fact $i \geq 5$, we also have 


$$
\begin{aligned}
f_{i+j} & =f_{i+j-1}+f\left(u_{i+j}\right) \leq f_{i+j-1}+f_{i+j-1}-f\left(u_{i}\right) \\
& \leq 2\left(3 \cdot 2^{i+j-3}-2^{j-1}+2\right)-\left(3 \cdot 2^{i-3}-2^{i-(n+3)}\right) \\
& =3 \cdot 2^{i+j-2}-2^{j}-3 \cdot 2^{i-3}+2^{i-(n+3)}+4 \\
& \leq 3 \cdot 2^{i+j-2}-2^{j}-2^{i-3}-1 \\
& =3 \cdot 2^{i+j-2}-2^{(i+j)-i}-2^{(i+j)-(j+3)}-1 .
\end{aligned}
$$

Since $i+(j+3) \leq m+n+3 \leq 2 n+3$, either $i<n+2$ or $j+3<n+2$. This implies $f_{i+j} \leq 3 \cdot 2^{(i+j)-2}-2^{(i+j)-(n+2)}-1$ and we have a contradiction by (4) of Proposition 3.4.

On the other hand, if $f\left(u_{i+j+1}\right) \leq f\left(u_{i}\right)+f\left(u_{i+j}\right)$, then by $(* *),\left(* *^{\prime}\right)$ and Lemma 2.3,

$$
\begin{aligned}
f_{i+j+1} & =f_{i+j-1}+f\left(u_{i+j}\right)+f\left(u_{i+j+1}\right) \leq f_{i+j-1}+f\left(u_{i+j}\right)+\left[f\left(u_{i}\right)+f\left(u_{i+j}\right)\right] \\
& \leq f_{i+j-1}+2\left(f_{i+j-1}+1\right)+f\left(u_{i}\right)=3 f_{i+j-1}+f\left(u_{i}\right)+2 . \\
& \leq 3\left(3 \cdot 2^{i+j-3}-2^{j-1}+2\right)+3 \cdot 2^{i-3}+3 \\
& =9 \cdot 2^{i+j-3}-3 \cdot 2^{j-1}+3 \cdot 2^{i-3}+9 .
\end{aligned}
$$

Since $i \geq 5$ and $j \geq 2$, we have $2^{i+j-3} \geq-3 \cdot 2^{j-1}+10$ and $2^{i+j-3} \geq 3 \cdot 2^{i-3}$. This implies $f_{i+j+1} \leq 11 \cdot 2^{i+j-3}-1 \leq 3 \cdot 2^{(i+j+1)-2}-2^{(i+j+1)-(n+2)}-1$. Again, this is not possible. Hence, we have the claim $i=\max \left\{k \mid v_{k} \in V_{2}\right\}$.

Now, since $i=\max \left\{k \mid u_{k} \in V_{2}\right\}$, we have $i-4 \geq t \leq n-2$ and $r_{\ell-4} \leq 0$ provided that $u_{\ell} \in V_{2}$ and $\ell \neq i$ in $\left(*^{\prime \prime}\right)$. By Lemma 2.5 and $\left(*^{\prime \prime}\right)$, we have

$$
\begin{aligned}
& f(G) \leq 2^{m+n-4} f_{4}+\sum_{j=1}^{t-1} 2^{m+n-4-j} \cdot 0+2^{m+n-4-t} \cdot 1+\sum_{j=t+1}^{m+n-4} 2^{m+n-4-j} \cdot(-2) \\
& =2^{m+n-4} \cdot 12+2^{m+n-4-t}-2\left(2^{m+n-4-t}-1\right)=3 \cdot 2^{m+n-2}-2^{m+n-4-t}+2 \\
& \leq 3 \cdot 2^{m+n-2}-2^{m+n-4-(n-2)}+2=3 \cdot 2^{m+n-2}-2^{m-2}+2 .
\end{aligned}
$$

Case 2. $f\left(u_{4}\right)=7$.

Review that $V_{1}=\left\{v \in V(G) \backslash V(H) \mid v u_{2} \notin E(G)\right\}$ and $V_{2}=\left\{v \in V(G) \backslash V(H) \mid v u_{4} \notin\right.$ $E(G)\}$. Clearly, $f_{4}=13$. Now, if $V_{2}=\emptyset$, then $r_{\ell} \leq-2$ for each $\ell \in[1, m+n-4]$ in $\left(*^{\prime \prime}\right)$. By Lemma 2.5 and $\left(*^{\prime \prime}\right)$, we have

$$
\begin{aligned}
f(G) & \leq 2^{m+n-4} f_{4}+\sum_{j=1}^{m+n-4} 2^{m+n-4-j}(-2)=13 \cdot 2^{m+n-4}-2\left(2^{m+n-4}-1\right) \\
& \leq 3 \cdot 2^{m+n-2}-2^{m-2}+2 .
\end{aligned}
$$


Otherwise, $V_{2} \neq \emptyset$. Let $i=\min \left\{j \mid u_{j} \in V_{2}\right\}$. Clearly $i \geq 5$. Since $u_{k} \in V_{1}$ for $k \in[5, i-1]$, $r_{k-4} \leq-2$ for $k \in[5, i-1]$ in $\left(*^{\prime}\right)$. By Lemma 2.5 and $\left(*^{\prime}\right)$, we have

$$
f_{i-1}=f_{4+(i-5)} \leq 2^{i-5} f_{4}+\sum_{j=1}^{i-5} 2^{i-5-j}(-2)=11 \cdot 2^{i-5}+2 .
$$

If $m+n=5$, then by Lemma 2.4, $f\left(u_{5}\right) \leq f_{4}-f\left(u_{4}\right)=13-7=6<f\left(u_{4}\right)$, which is impossible. Hence, consider $m+n \geq 6$. Again, by Lemma 2.4, either $f\left(u_{i}\right) \leq f_{i-1}-f\left(u_{4}\right)$ or $f\left(u_{i+1}\right) \leq f\left(u_{i}\right)+f\left(u_{4}\right)$ when $i+1 \leq m+n$. First, if $f\left(u_{i}\right) \leq f_{i-1}-f\left(u_{4}\right)$, then $f_{i}=f_{i-1}+f\left(u_{i}\right) \leq 2 f_{i-1}-f\left(u_{4}\right)$. By $(*)$ and $(* * *)$,

$$
\begin{aligned}
& f(G)<2^{m+n-i}\left(f_{i}+1\right) \leq 2^{m+n-i}\left[2 f_{i-1}-f\left(u_{4}\right)+1\right] \leq 2^{m+n-i}\left[11 \cdot 2^{i-4}-2\right] \\
& \leq 11 \cdot 2^{m+n-4} \leq 3 \cdot 2^{m+n-2}-2^{m-2}+2 .
\end{aligned}
$$

On the other hand, if $f\left(u_{i+1}\right) \leq f\left(u_{i}\right)+f\left(u_{4}\right)$, then by $(* * *)$, we have

$$
\begin{aligned}
& f_{i+1}=f_{i-1}+f\left(u_{i}\right)+f\left(u_{i+1}\right) \leq f_{i-1}+f\left(u_{i}\right)+\left[f\left(u_{i}\right)+f\left(u_{4}\right)\right] \\
& \leq f_{i-1}+2\left(f_{i-1}+1\right)+f\left(u_{4}\right)=33 \cdot 2^{i-5}+15
\end{aligned}
$$

If $i \geq 6$, then by $(*)$, we have

$$
\begin{aligned}
& f(G)<2^{m+n-(i+1)}\left(f_{i+1}+1\right) \leq 2^{m+n-(i+1)}\left(33 \cdot 2^{i-5}+16\right) \\
& =33 \cdot 2^{m+n-6}+16 \cdot 2^{m+n-(i+1)} \leq 33 \cdot 2^{m+n-6}+16 \cdot 2^{m+n-7} \\
& =41 \cdot 2^{m+n-6}<3 \cdot 2^{m+n-2}-2^{m-2}+2 .
\end{aligned}
$$

Therefore, the case left to check is that $i=5$. First, we evaluate $f\left(u_{6}\right)$ and $f_{6}$. By assumption and Lemma 2.3, $f\left(u_{6}\right) \leq f\left(u_{5}\right)+f\left(u_{4}\right) \leq\left(f_{4}+1\right)+f\left(u_{4}\right)=21$, and $f_{6}=f_{4}+f\left(u_{5}\right)+f\left(u_{6}\right) \leq f_{4}+\left(f_{4}+1\right)+f\left(u_{6}\right) \leq 48$. Now, if there exist a $k^{\prime} \geq 7$ such that $u_{k^{\prime}} \in V_{2}$. Let $\ell=\min \left\{j \geq 7 \mid u_{j} \in V_{2}\right\}$. Then for each $k \in[7, \ell-1], u_{k} \in V_{1}$ and hence $r_{k-6} \leq-2$ in $\left(*^{\prime}\right)$. By Lemma 2.5 and $\left(*^{\prime}\right)$, we have

$$
f_{\ell-1} \leq 2^{\ell-7} f_{6}+\sum_{j=1}^{\ell-7} 2^{\ell-7-j}(-2) \leq 46 \cdot 2^{\ell-7}+2 .
$$

Also, by Lemma 2.4, either $f\left(u_{\ell}\right) \leq f_{\ell-1}-f\left(u_{4}\right)$ or $f\left(u_{\ell+1}\right) \leq f\left(u_{\ell}\right)+f\left(u_{4}\right)$ when $\ell+1 \leq m+n$. First, if $f\left(u_{\ell}\right) \leq f_{\ell-1}-f\left(u_{4}\right)$, then

$$
\begin{aligned}
& f_{\ell}=f_{\ell-1}+f\left(u_{\ell}\right) \leq 2 f_{\ell-1}-f\left(u_{4}\right) \leq 92 \cdot 2^{\ell-7}-3 \\
& \leq 96 \cdot 2^{\ell-7}-2^{\ell-5}-3 \leq 3 \cdot 2^{\ell-2}-2^{\ell-(n+2)}-1 .
\end{aligned}
$$


This is a contradiction. On the other hand, if $f\left(u_{\ell+1}\right) \leq f\left(u_{\ell}\right)+f\left(u_{4}\right)$, then since $\ell \geq 7$, we have

$$
\begin{aligned}
f_{\ell+1} & =f_{\ell-1}+f\left(u_{\ell}\right)+f\left(u_{\ell+1}\right) \leq f_{\ell-1}+2 f\left(u_{\ell}\right)+f\left(u_{4}\right) \\
& \leq f_{\ell-1}+2\left(f_{\ell-1}+1\right)+f\left(u_{4}\right) \leq 3 \cdot\left(46 \cdot 2^{\ell-7}+2\right)+9 \\
& =69 \cdot 2^{\ell-6}+8 \cdot 2-1 \leq 77 \cdot 2^{\ell-6}-1 \\
& \leq 96 \cdot 2^{\ell-6}-16 \cdot 2^{\ell-6}-1 \leq 3 \cdot 2^{(\ell+1)-2}-2^{(\ell+1)-(n+2)}-1 .
\end{aligned}
$$

We also have a contradiction.

This implies that $u_{k} \in V_{1}$ for each $k \in[7, m+n]$. Therefore, $r_{k-6} \leq-2$ for each $k \in[7, m+n]$ in $\left(*^{\prime}\right)$. By Lemma 2.5 and $\left(*^{\prime}\right)$, we have

$$
\begin{aligned}
& f(G)=f_{6+(m+n-6)} \leq 2^{m+n-6} f_{6}+\sum_{j=1}^{m+n-6} 2^{m+n-6-j}(-2) \\
& \leq 48 \cdot 2^{m+n-6}-2\left(2^{m+n-6}-1\right) \leq 3 \cdot 2^{m+n-2}-2^{m-2}+2 .
\end{aligned}
$$

This concludes the proof of the theorem.

\section{References}

[1] R. Alter, J. A. Brnett, A postage stamp problem, Amer. Math. Monthly 87(1980) 206-210.

[2] N. G. de Bruijn, On base for the set of integers, Publ. Math. (Debrecen)1(1950) $232-242$.

[3] J. F. Fink, Labelings that realize connected subgraphs of all conceivable values, Congressus Numerantium 132(1998) 29-37.

[4] J. A. Gallian, A survey: recent results, conjectures and open problems in labelling graphs, J. Graph Th. 13(1989) 491-504.

[5] R. L. Graham, N. Sloane, On additive bases and harmonious graphs, SIAM J. Algebraic Discrete Math. 1(1980) 382-404.

[6] R. Guy, The Postage Stamp Problem, Unsolved Problems in Number Theory, second ed., Springer, New York, 1994, pp. 123-127.

[7] R. L. Heimer, H. Langenbach, The stamp problem, J. Recreational Math. 7(1974) 235-250.

[8] C. T. Long, N. Woo, On bases for the set of integers, Duke Math. J. 38(1971) 583-590.

[9] W. F. Lunnon, A postage stamp problem, Comput. J. 12(1969) 377-380.

[10] L. Moser, On the presentation of 1, 2,.., $n$ by sums, Acta Arith. 6(1960) 11-13. 
[11] S. Mossige, The postage problem: an algorithm to determine the $h$-range of the $h$ range formula on the extremal basis problem for $k=4$, Math. Comput. 69(2000) 325-337.

[12] S. G. Penrice, Some new graph labeling problems: a preliminary report, DIMACS Tech. Rep. 95-26(1995) 1-9.

[13] E. Salehi, Sin-Min Lee, M. Khatirinejad, IC-colorings and IC-indices of graphs, Discrete Math. 299(2005) 297-310.

[14] E. S. Selmer, On the postage stamp problem with the three stamp denominations, Math. Scand. 47(1980) 29-71.

[15] R. G. Stanton, J. G. Kalbfleisch, R. C. Mullin, Some tables for the postage stamp problem, Proceedings of 4th Manitoba Conference on Numerical Math., Utilitas Math. Pub., Winnipeg, 1974, 351-356.

[16] A. Stöhr, Gelöste und ungelöste Fragen uber Basen der naturlichen Zahlenreihe I, II, J. Reine Angew. Math. 194(1955) 111-140.

[17] D. B. West, Introduction to graph theory, second edition, Prentice-Hall, 2001. 